\title{
REPEATEDLY APPENDING ANY DIGIT TO GENERATE COMPOSITE NUMBERS
}

\author{
JON GRANTHAM, WITOLD JARNICKI, JOHN RICKERT, AND STAN WAGON
}

\begin{abstract}
We investigate the problem of finding integers $k$ such that appending any number of copies of the base-ten digit $d$ to $k$ yields a composite number. In particular, we prove that there exist infinitely many integers coprime to all digits such that repeatedly appending any digit yields a composite number.
\end{abstract}

\section{INTRODUCTION}

Recently Lenny Jones [1] asked about integers that yield only composites when a sequence of the same base-ten digit is appended to the right. He showed that 37 is the smallest number with this property when appending the digit $d=1$. For each digit $d \in\{3,7,9\}$, he also found numbers coprime to $d$ that yield only composites upon appending $d s$.

In this paper we find a single integer that works for all digits simultaneously. More precisely,

Theorem. There are infinitely many positive integers $k$ with $\operatorname{gcd}(k, 2 \cdot 3 \cdot 5 \cdot 7)=$ 1 , such that for any base-ten digit d, appending any number of $d s$ to $k$ yields a composite number.

Further, we will investigate the question of the smallest numbers that remain composite upon appending strings of a digit for each particular digit. Jones found, for digits $3,7,9$, respectively, the examples 4070,606474 , and 1879711 . It appears that 4070 is the smallest for $d=3$; for digit 7 , we found 891 , which is almost certainly minimal; and for digit 9 , the likely answer 10175 was discovered by [9]. In the next section, we explain the obstructions to proving that these three answers are the smallest.

\section{SEEDS}

Given a digit $d$, let's use the term seed for a number coprime to $d$ such that appending any number of $d$ s on the right yields a composite. The smallest positive integer with this property will be referred to as a minimal seed. Only the cases $d \in\{1,3,7,9\}$ are nontrivial. Jones proved that 37 is the minimal seed for $d=1$, and he also found the seed 4070 for digit 3 . For every $k<4070$, except 817 , we have found a value of $n$ such that appending $n$ 3s yields a prime or, in three cases, a probable prime. For 817, appending up to 554789 3s yielded only composites. But factorizations show no apparent obstruction to primality, so we conjecture that 4070 is the minimal seed for digit 3.

A covering congruence argument shows that 891 is a seed for digit 7 . It is easy to see that the number obtained by appending $6 m+i 7 \mathrm{~s}$ to 891 is divisible by 3 , 
$37,11,3,1113$, respectively, as $i$ varies from 0 to 5 . We have shown that 891 is a minimal seed, under the assumption that appending $113307 \mathrm{~s}$ to 480 , and 28895 $7 \mathrm{~s}$ to 851 yields primes. Each of these two large numbers has passed 200 strong pseudoprime tests. For all other potential seeds below 891, we have found primes that can be certified using elliptic curve methods with Mathematica or Primo [4]. We used Primo on the largest cases; the largest was $9777 \ldots 7$ with $29047 \mathrm{~s}$, which took 45 hours.

The digit-9 case asks for an integer $k$ such that $(k+1) 10^{n}-1$ is always composite; it is thus a variation on the classic Riesel problem [3, 6, 7, 8], which addresses the same question in base 2. For that classic case, it is known that 509201 works, meaning that $509202 \cdot 2^{n}-1$ is composite for $n \geq 0$. Participants in the Riesel project have also investigated the decimal case, and showed [9] that the expected minimal seed for digit 9 is 10175 . To see that this is a seed we again consider the number of appended digits modulo 6 and use the cover $\{11,7,11,37,11,13\}$. Of the smaller numbers, only two, 4420 and 7018, have not been eliminated as seeds. The Riesel project $[7,8]$ has checked each through the addition of $6300009 \mathrm{~s}$ without finding a prime. In this case, primality proving for a probable prime is easy using the Lucas $n+1$ test.

\section{A PANDigital SEed}

It is not hard to find an integer that remains composite when any sequence of the form $d d d \ldots d$ is appended on the right, where $d$ is any decimal digit. We leave it as an exercise to show that 6930 does the job; only the case $d=1$ requires a prime cover, and the one used in $\S 2$ for 891 works. Some prime searching shows that 6930 is the smallest such example (the most difficult candidate to eliminate was $6069 ; 15251$ s yielded a prime).

A more natural problem in our context is to consider only the digits 1, 3, 7, 9 and ask for an integer $k$ that is a seed for each of these four digits (thus $k$ is coprime to 3 and 7 ). We call such a positive integer $k$ a pandigital seed.

Theorem. A pandigital seed exists. An example is 4942768284976776320.

Proof. A proof requires only checking that certain covers work, but we outline the method by which the large seed and corresponding covers were found. We first need to analyze the condition that a term in the sequence is divisible by a given prime $p$. Appending $n$ copies of the digit $d$ to the integer $k$ yields the value

$$
s_{n}:=k \cdot 10^{n}+\frac{d\left(10^{n}-1\right)}{9} .
$$

If we assume that $p \notin\{2,3,5\}$, then $s_{n} \equiv 0(\bmod p)$ if and only if

$$
9 k \cdot 10^{n}+\frac{d\left(10^{n}-1\right)}{9},
$$

which is equivalent to

$$
k \equiv 9^{-1} d\left(10^{-n}-1\right) \quad(\bmod p) .
$$

If $p=3$, then we instead have the condition

$$
s_{n} \equiv k+d \frac{\left(10^{n}-1\right)}{9} \equiv 0 \quad(\bmod 3)
$$




\begin{tabular}{|l||c|c|c|c|}
\hline & $n \equiv 1(\bmod 6)$ & $n \equiv 5(\bmod 6)$ & $n \equiv 1(\bmod 6)$ & $n \equiv 5(\bmod 6)$ \\
\hline digit 1 & $k \equiv 2(\bmod 7)$ & $k \equiv 1(\bmod 7)$ & $k \equiv 9(\bmod 13)$ & $k \equiv 1(\bmod 13)$ \\
digit 3 & $k \equiv 6(\bmod 7)$ & $k \equiv 3(\bmod 7)$ & $k \equiv 1(\bmod 13)$ & $k \equiv 3(\bmod 13)$ \\
digit 7 & $k \equiv 0(\bmod 7)$ & $k \equiv 0(\bmod 7)$ & $k \equiv 11(\bmod 13)$ & $k \equiv 7(\bmod 13)$ \\
digit 9 & $k \equiv 4(\bmod 7)$ & $k \equiv 2(\bmod 7)$ & $k \equiv 3(\bmod 13)$ & $k \equiv 9(\bmod 13)$ \\
\hline
\end{tabular}

TABLE 1. Conditions on $k$ to guarantee that 7 or 13 divides the number obtained by appending a digit string to $k$.

which reduces to $k \equiv 2 d n(\bmod 3)$. It is useful to observe that because $10^{n} \equiv 1$ $(\bmod 11)$, when $n$ is even, so in this case we have that $s_{n}$ is congruent modulo 11 to the seed itself. Therefore $k \equiv 0(\bmod 11)$ is a helpful condition. Hence, we may focus on odd values of $n$.

Recall the cover $\{11,7,11,37,11,13\}$ used to prove that 10175 is a seed for digit 9. For that digit we may use (1) and note the positions of 7, 13, and 37 in the cover (which correspond to odd values of to learn that divisibility by these three primes in the corresponding modulo- 6 cases requires $k \equiv 4(\bmod 7), 9(\bmod 13)$ and 0 $(\bmod 37)$, respectively. For example, when $p=37$ and the number of appended digits is $n=6 i+3$, equation (1) gives

$$
k \equiv 10^{-(6 i+3)}-1 \equiv\left(10^{-6}\right)^{i} 10^{-3}-1 \equiv 0 \quad(\bmod 37) .
$$

But if we insist that $k \equiv 9(\bmod 13)$, then it is easy to see that $(1)$ is impossible when $d=3$ or 7 . So, 13 cannot be used in a cover for these digits.

In practice, though, it is more useful to begin with the primes 11 and 37. Since 11 and 37 have such small periods (the order of 10 modulo these primes is 2 and 3 , respectively), it is not surprising that the only overlap between digits is the residue 0 . For example, the solutions to (1) modulo 11 are $k=0, d(\bmod 11)$. Choosing $k \equiv d(\bmod 11)$ covers residue classes only for the digit $d$ - leaving $7 / 8$ of the sequence values to be covered by other primes. Choosing $k \equiv 0(\bmod 11)$ means that half of the cases for each digit are covered, leaving only half the terms of the sequences to be covered by other primes. Similarly adding the condition $k \equiv 0$ (mod 37) leaves only one-third of the sequences' values to be covered by other primes, specifically the values with $6 i+1$ added digits, and the values with $6 i+5$ added digits. Since the primes 7 and 13 each cover one-sixth of the residues, these can be checked to see how many of the covers can be completed. For each digit we determine $k$ modulo 7 and modulo 13 for these two cases. This gives the conditions in Table 1.

Adding the condition $k \equiv 1(\bmod 10)$ leads to the seed 1970728582053685108721 , which has the property that it is coprime to each of the 10 digits and appending any string of identical digits leads to a composite.

This means that if $k \equiv 1,3$, or $9(\bmod 13)$, then two of the remaining eight cases are divisible by 13. A modulo-7 condition takes care of another case or two. Then we must find some other primes to get compositeness in the remaining cases. Some searching led to the following four coherent covers, using the 13 primes $3,7,11,13,31,37,41,73,101,137,211,241,271$. The periods for the four cases are $6,6,30$, and 8 , respectively. 


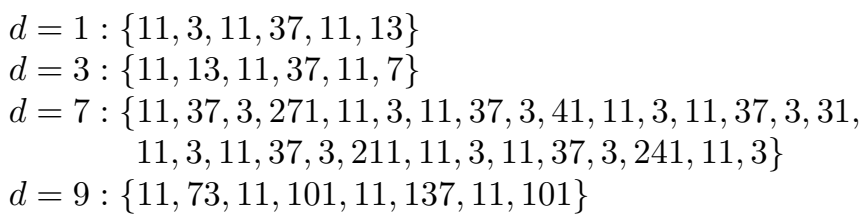

These four covers are coherent in the sense that each prime for each digit leads, by (1), to a congruence condition on $k$ (see Table 2), and those conditions are consistent. The Chinese remainder theorem applied to the 13 congruences in Table 2 yields 4942768284976776320 , which is therefore a pandigital seed in base ten.

If we add $k \equiv 1(\bmod 210)$ to the list in Table 2 , we obtain infinitely many values of $k$ satisfying the theorem as stated in $\S 1$.

\begin{tabular}{|l||l|l|l|l|}
\hline digit 1: & $0(\bmod 11)$ & $0(\bmod 37)$ & $1(\bmod 13)$ & $2(\bmod 3)$ \\
\hline digit 3: & $0(\bmod 11)$ & $0(\bmod 37)$ & $1(\bmod 13)$ & $3(\bmod 7)$ \\
\hline \multirow{2}{*}{ digit 7: } & $0(\bmod 11)$ & $0(\bmod 37)$ & $20(\bmod 31)$ & $2(\bmod 3)$ \\
& $0(\bmod 271)$ & $28(\bmod 41)$ & $106(\bmod 211)$ & $7(\bmod 241)$ \\
\hline digit 9: & $0(\bmod 11)$ & $9(\bmod 101)$ & $40(\bmod 137)$ & $21(\bmod 73)$ \\
\hline
\end{tabular}

TABLE 2. Congruence conditions on $k$ derived from four coherent covers that lead to the pandigital seed $k=4942768284976776320$

\section{Open PROBLEMS}

We conclude with some unsolved problems.

(1) Find a number of 3 s which can be appended to 817 to obtain a probable prime, thus completing the proof, modulo probable primes, that 4070 is minimal for the digit 3 .

(2) Find a number of $9 \mathrm{~s}$ which can be appended to 4420 or 7018 to produce a prime.

(3) Certify primality of 480 with $113307 \mathrm{~s}$ appended and 851 with $288957 \mathrm{~s}$. Doing so would complete the digit-7 case.

(4) Data for all bases up to 10 can be found at [5]. Similar problems exist for these bases.

(5) Find a base-ten pandigital seed that is smaller than 4942768284976776320.

(6) Investigate for various bases the situation where the appended digits come from a fixed sequence, as was done by Jones and White [2] for base ten.

\section{REFERENCES}

[1] Lenny Jones. When does appending the same digit repeatedly on the right of a positive integer generate a sequence of composite integers? Amer. Math. Monthly, 118(2):153-160, 2011.

[2] Lenny Jones and Daniel White. Appending digits to generate an infinite sequence of composite numbers. J. Integer Seq., 14(5):Article 11.5.7, 12, 2011.

[3] Wilfrid Keller. The Riesel problem: Definition and status. http://www.prothsearch.net/ rieselprob.html.

[4] Marcel Martin. Primo. http://www.ellipsa.eu, 2010. Version 3.0.9.

[5] John Rickert. Composite sequences. http://www.rose-hulman.edu/ rickert/Compositeseq. 
[6] H. Riesel. Några stora primtal. Elementa, 39:258-260, 1956.

[7] Riesel conjectures and proofs. http://www.noprimeleftbehind.net/crus/ Riesel-conjectures.htm.

[8] The Riesel problem. http://www.primegrid.com/forum_thread.php?id=1731\&nowrap=true\# 21625.

[9] Mark Rodenkirch. Sierpinski/Riesel base 10. http://www.mersenneforum.org/showthread. php?t=6911, January 2007 .

Institute for Defense Analyses, Center for Computing Sciences, Bowie, Maryland, USA

E-mail address: grantham@super.org

Google Kraków, Kraków, Poland

E-mail address: witoldjarnicki@google.com

Rose-Hulman Institute of Technology, Terre Haute, Indiana, USA

E-mail address: rickert@rose-hulman.edu

Macalester College, St. Paul, Minnesota, USA

E-mail address: wagon@macalester.edu 